Original Research Paper

\title{
Development of a Dashboard for a Local Food Bank
}

\author{
Yogeeta Desai, Steven Jiang and Lauren Davis \\ Department of Industrial and Systems Engineering, North Carolina A\&T State University, Greensboro, USA
}

\author{
Article history \\ Received: 06-03-2017 \\ Revised: 09-03-2017 \\ Accepted: 16-03-2017 \\ Corresponding Author: \\ Yogeeta Desai \\ Department of Industrial and \\ Systems Engineering, North \\ Carolina A\&T State University, \\ Greensboro, USA \\ Email: desaiyogeeta@yahoo.com
}

\begin{abstract}
Hunger relief is one of the major needs during humanitarian emergencies. It presents significant challenges to aid organizations trying to manage data, information and knowledge about the situation or event. Food banks receive donations from a variety of sources to meet their demand. The distribution of donated food to meet the unmet hunger needs is a critical issue faced by the food banks across the nation. This paper presents a methodology to apply interactive dashboards to a food bank's decision making process. The first step of this research focuses on understanding the existing decision making process of a food bank. Data from a local food bank was used for this research. Data mining tools were employed to develop predictive models for food bank decision making. Appropriate visualization techniques were identified that can be used to visualize the data mining results. An interactive dashboard was developed and evaluated to enable effective decision making. The results indicated that interactive dashboards were highly effective as compared to the traditional data retrieval system for decision making in food bank operations. The new methodology can be extended to other food banks and hunger-relief organizations which deal with big data.
\end{abstract}

Keywords: Visual Analytics, Humanitarian Relief, Interactive Dashboard, Food Bank

\section{Introduction}

An estimated $14 \%$ of households in the United States were food insecure at least once during the year 2014 (Coleman-Jensen et al., 2015). Food insecurity means that households had a shortage of food for an active, healthy life for one or more of their members. In addition, 5.6\% of households had very low food security which meant that their eating patterns were disrupted because of the lack of money and other resources for food (Gundersen et al., 2011). Studies have shown that most food insecurity is associated with chronic poverty and temporary unemployment (Barrett, 2010). On the other hand, American families waste approximately $25 \%$ of the food and beverages they buy (Bloom, 2011). Reducing food losses by just 15\% would be enough to feed more than 25 million Americans every year when one in six Americans lack a secure supply of food to their tables (Coleman-Jensen et al., 2011). Increasing the efficiency of the food system is a triple bottom-line solution that requires collaborative efforts by businesses, governments and consumers (Coleman-Jensen et al., 2011).
One of the nation's largest hunger-relief organizations, Feeding America assists local food banks in acquiring and dispensing food, raising funds and acquiring more donors, sharing best practices amongst food banks and other agencies. Food banks receive donations from a variety of sources to meet their demand. However, the donations received may not be efficiently translated into food provision for the hungry due to several shortcomings. Some of the reasons include (1). Uncertain donation and demand patterns, (2). Untimely logistical needs to deliver food to the hungry population, (3). Limited information on the local distribution and manpower needs to distribute food and (4). Unavailability of a structured information tool for decision making at different levels in the food bank hierarchy. Thus, it is imperative that a systematic methodology be developed that can assist food bank administrators make informed decisions on food and resource allocations. In addition, it is important to be able to predict food demand accurately and investigate the critical factors that impact food demand.

In recent years, data mining has been applied to develop an empirical model to investigate various factors 
that affect food demand for a food bank (Okore-Hanson, 2012). A logistic regression model was developed to predict whether a person will donate or not to a humanitarian agency during natural disasters. Brock and Davis (2015) used neural network models to forecast inkind donations from retail donors for a food bank. Nuamah et al. (2015) have developed a state-space based simulation model for exponential smoothing to determine the expected quantity of food donations received per month in a multi-warehouse distribution network. The donation data for the food bank was analyzed using time series models based on product types, branch and program types (Terry, 2013). Though, several approaches to analyzing food bank data have been conducted, a systems level approach needs further investigation. Further, the visualization of the food bank data in a real-time system for decision making is missing.

Visual analytics (VA) is a combination of automated analysis techniques with interactive visualizations for an effective understanding, reasoning and decision making for large and complex data sets (Keim et al., 2008). Andrienko et al. (2007) have developed a visual analytics software module to support planning of massive transportations under time-critical conditions, in particular evacuation of people from a disaster-affected area. Arias-Hernandez and Fisher (2013), present a qualitative methodology for design research in the domain of visual analytics for emergency management. In recent years, the International Organization for Migration (IOM) has used $\mathrm{SAS}^{\circledR}$ data visualization and text mining to identify and rally responders to assist with the most critical problems in areas devastated by super Typhoon Haiyan (SAS, 2015). The application of VA can uncover hidden data trends that would go unnoticed due to lack of visualization techniques. Achieving this goal requires advanced analytics, including forecasting and optimization, as well as integrated data management with visualization tools.

In this research, interactive dashboards were developed using a user centered approach. Visual Analytics (VA) approach was used for developing interactive dashboards. Visual analytics software Tableau $^{\circledR}$ was used to develop the interactive dashboards. These dashboards were evaluated for their effectiveness and ease of use with subject matter experts (food bank personnel) and user participants.

\section{Methodology}

Interactive dashboards were developed using visual analytics which is composed of data analytics, visualization and interactivity. Data analytics models were built using supervised and unsupervised learning methods. Time series models were developed to forecast distribution trends for the food bank. Different visualization tools were selected based on a detailed literature review provided below. User centered design approach was used for developing interactive dashboards. Further, interactivity modes applicable to food bank data were evaluated by the participants. The dashboard was evaluated by subject matter experts (food bank personnel) and user participants. Statistical analysis including descriptive and inferential statistics were used to evaluate the dashboard design based on time to completion of a task and overall satisfaction. The final dashboard interface was developed using the above described inputs. This paper focuses the on the development of the interactive dashboard to visualize the results of the data analytics.

\section{Visualization Tool Selection}

A detailed literature review was conducted to justify the use of different visualization and interactive tools to be implemented in this research. The following are examples of visualization tools for display of the food bank data: Pie-charts, Line charts, Bar charts.

Pie charts should be used when visual representation of discontinuous variable (discrete or nominal) is desired (Annesley, 2010). Briefel et al. (2003) have used pie charts to visualize the food insecurity and frequencies of visits to different Emergency Food Assistance System (EFAS) providers. Line charts have been used to show prevalence of food insecurity and very low food security in U.S. households over the years (Coleman-Jensen et al., 2015). Barrett (2010) uses a line chart to show how different food security proxy indicators paint different pictures (Barrett, 2010). Taking insight from literature sources, line charts have been utilized to display fluctuations in foodbank resources with respect to time. Bar charts have been used to visualize EFAS data to show the percentage of different EFAS provider who distribute USDA food commodities (Briefel et al., 2003). Further, bar chart has been used to visualize delays and cancellation of surgical procedures for different specialties making it easy to understand the data (Jonnalagadda et al., 2005). In this research, bar charts have been used to display different food types, branch types and donation types.

\section{Dashboard Development Using User Centered Design}

Regular monitoring of data is critical for effective decision making of the food bank. Dashboards are used to show key performance indicators of an organization. The interactive dashboard has been built using a User Centered Design (UCD) approach. The following iterative five-step process was employed to build the interactive dashboard.

The UCD focuses on user requirements in the initial stages of product development to ensure completeness and satisfaction of the final interface design. To achieve 
effective, efficient and usable interfaces the user's perspective needs to be included through all the stages of the design and development process (Adams, 2002). The UCD approach involves multidisciplinary design teams which leverage user feedback during the planning, design and evaluation phases of system design (Wickens et al., 1998). This concept ensures that the system is designed from a user perspective versus designs in which the user has to adapt to the design (Vredenberf et al., 2001). The basic UCD methodology follows an iterative five-step process which includes: 1 . User Profiles, 2. Task Analysis, 3. Prototype, 4. Usability Evaluation, 5. Final Interface. In this research, the UCD steps were implemented for the dashboard development as described below.

\section{User Profiles}

The first phase was identifying the food bank users of the dashboard. It involved data collection related to the needs and expectations of the users. In the initial phase five food bank personnel were interviewed with a questionnaire designed to extract information about their decision making process. The local food bank involved in this research was Food bank of central and eastern North Carolina (FBCENC). The key stakeholders for this research were the Vice president of operations, FBCENC managers and data management staff. Foodbank data was used to develop the prototype interactive dashboard.

\section{Task Analysis}

The task analysis was carried out to identify and gain deeper understanding of the user tasks. The subject matter experts which include the Vice president of operations, IT manager and data management staff from FBCENC were interviewed. The primary tasks were discussed with them. FBCENC officials perform several tasks to obtain data for decision making. The most common task is to generate report for a given agency/branch. A hierarchical task analysis was performed for the existing process as shown in Fig. 1.

\section{Prototype}

In this phase, an interactive dashboard was developed based on inputs from the previous two steps. A prototype interface was created using Tableau ${ }^{\circledR}$ software. Key attributes derived during the interview and the task analysis were used as inputs to develop design guidelines for the dashboard. This prototype was used in the early dashboard testing trials. Five Subject Matter Experts (SME) were utilized as a pilot test group to screen for major problems before user testing. SMEs were encouraged to interact with the prototype to evaluate the usability. SME review comments were incorporated as a feedback for iterative improvement of the dashboard design. Based on the feedback from FBCENC personnel, a new process was developed for the task of generating a report for a given agency/branch. Figure 2 shows the task analysis for the new process.

\section{Usability Evaluation}

In this phase, usability testing was performed on the prototype interactive dashboard. The testing protocol was developed to conduct the study. Institutional Review Board (IRB) approvals were sought before running the experiment.

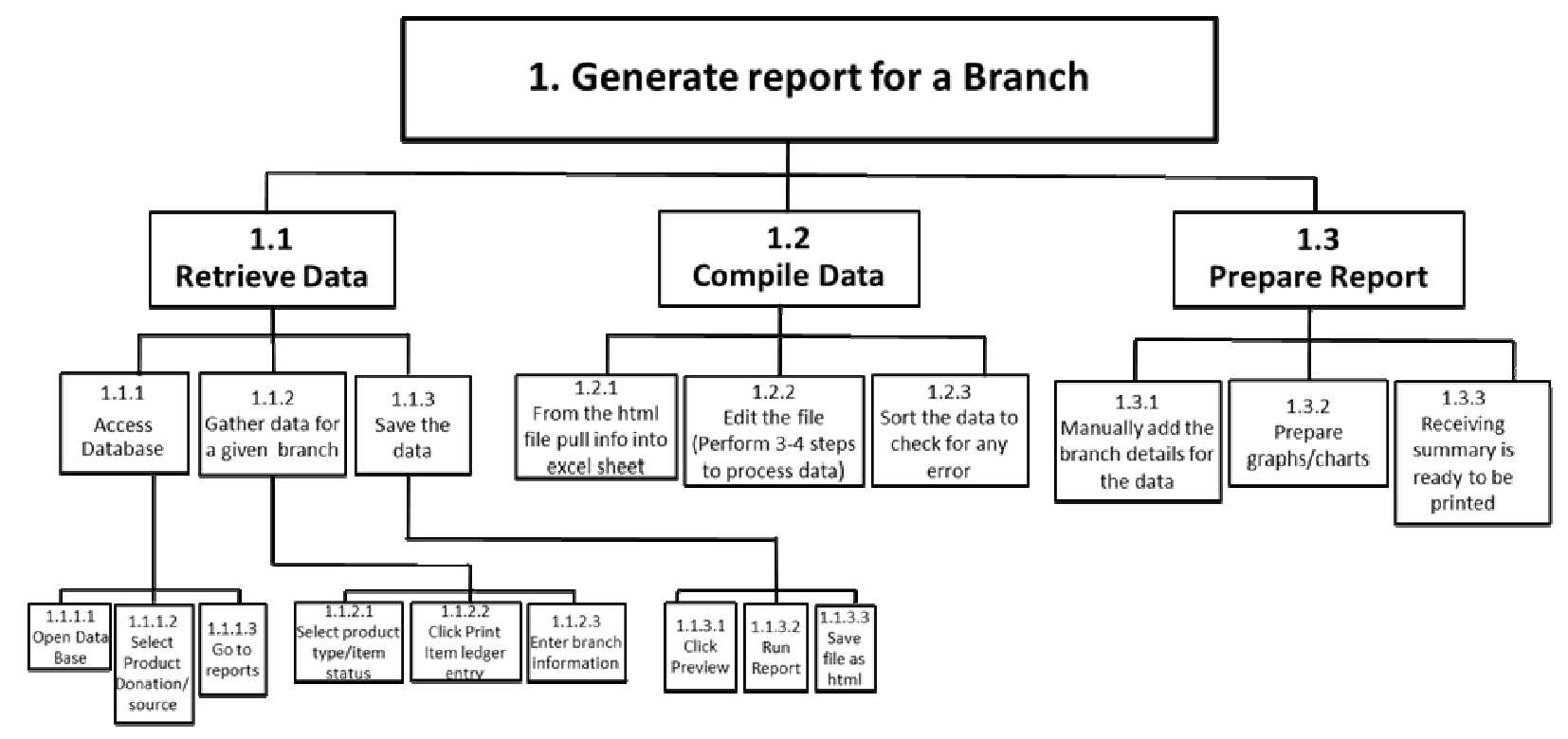

Fig. 1. Hierarchical task analysis for generating a report for a food bank branch 


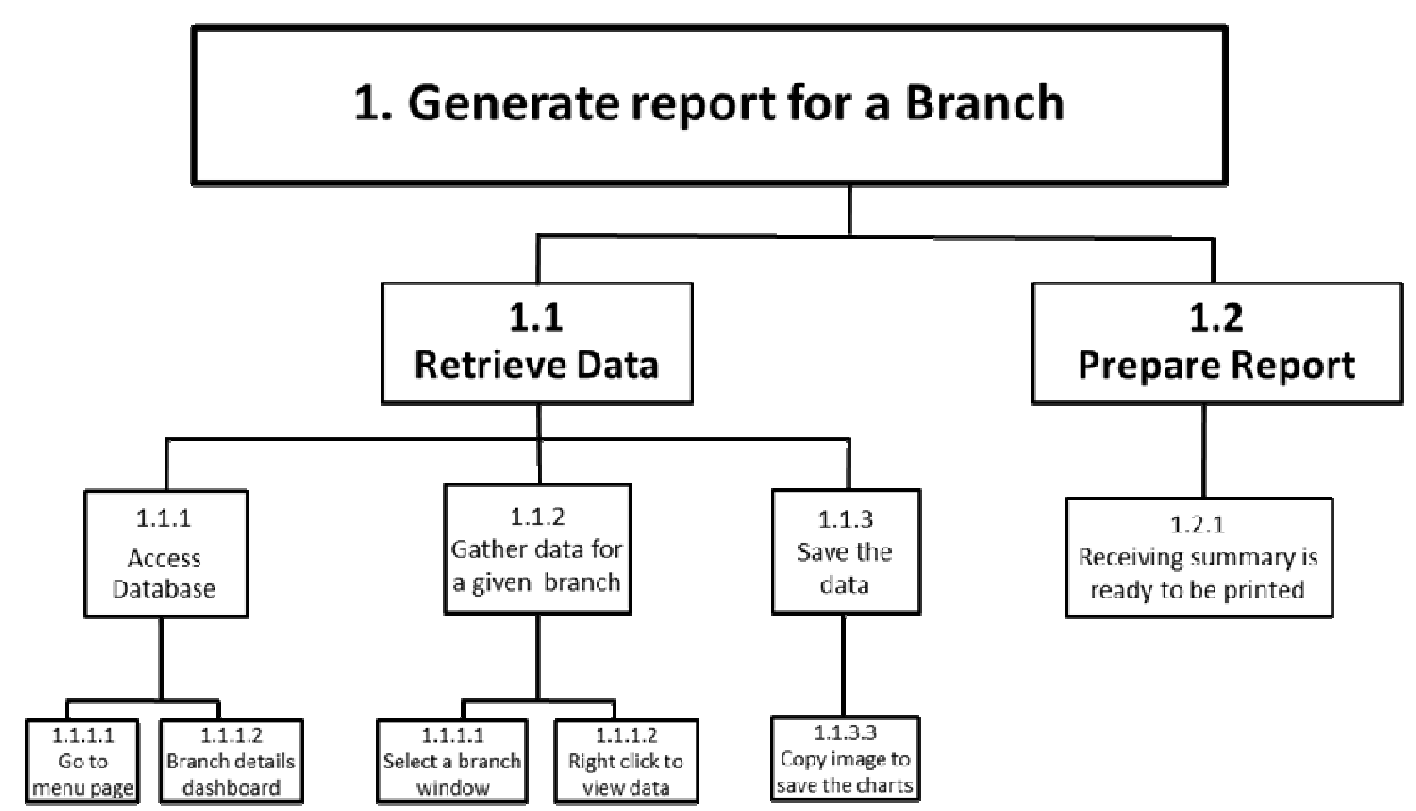

Fig. 2. Hierarchical task analysis for the new interactive dashboard design

\section{Participant}

Fifteen graduate and undergraduate students were recruited to evaluate the dashboard design. The details of the participant collected in a pre-trial survey are as follows. $33 \%$ of the participants had a proficiency level of "good" and $60 \%$ of the participants had a proficiency level of "very good" with computer usage and had prior experience with visualization tools. 13 out of the 15 participants had used data analytics with a high level of proficiency. For a total of 15 participants, $27 \%$ were undergraduate versus $73 \%$ were graduate students. $67 \%$ were male participants and $33 \%$ female participants. $40 \%$ of participants had an age group of 20-24 years, while the age groups of $25-29$ was $34 \%$ and $30-34$ had $20 \%$ of the participants, respectively.

\section{Stimulus Material}

Interactive dashboards built for this research were evaluated.

\section{Experimental Design}

A completely randomized design was used. Participants were randomly assigned to the two dashboards (existing vs. prototype dashboard) to be evaluated.

\section{Task}

The participants evaluated 3-4 different dashboards and answered related questions. One of the tasks the participants performed was to generate the report for the different branches.

\section{Procedure}

Participant were asked to complete a pre-test questionnaire to solicit background information and experience. The participants were given training on how to use the interactive dashboard. Then, he/she was asked to evaluate the interactive dashboards. The interactive dashboards consisted of different visualization charts (pie chart, bar chart, line chart etc.) used to show data from different branches for different entities over the years. Participants answered the questions related to the food bank data for different charts. After the dashboard evaluation participants filled the Post Study System Usability Questionnaire (PSSUQ) survey (US, 2015).

\section{Data Collection}

From the given set of tasks of evaluating the interactive dashboards, performance data of time and error was collected. Both subjective and objective measures were collected from the experiment. The subjective measure consisted of 16 question survey which was designed to test the overall dashboard design. The survey questions were divided into: (a) Ease of use, (b) information quality and (c) interface quality. The survey questions were rated on a Likert scale of 1 through 7. Multivariate analysis of variance was used to analyze the data. The objective measure includes the time to completion for the task.

\section{Implement}

Using the evaluation findings of the study, the dashboard was revised and given to FBCENC for potential implementation. 


\section{Results}

\section{Dashboard Development}

Based on discussion with the food bank Subject Matter Experts (SME) and studying the donation/distribution process an interactive dashboard was developed.

\section{Dashboard Prototype}

An initial version of the dashboard was developed based on the process evaluation and requirements analysis. Visualization tools such as bar charts, pie charts and line charts were selected based on their applicability towards food bank data as discussed in the methodology section. Figure 3 shows the initial version of the dashboard interface. It consists of the home page, FBCENC overview, FBCENC donation details, FBCENC distribution details and FBCENC branch details. The home page for the dashboard has four categories which will navigate to individual dashboards for specific details. The FBCENC Overview gives the overall information for the donation and distribution. The FBCENC Donation and Distribution Details dashboards give detailed information for each category based on product type, donation and distribution programs. The FBCENC Branch Details gives detailed information on a branch. Detail dashboard views and explanation for some of the dashboard categories are described in this section.

\section{FBCENC Overview Dashboard}

Figure 4 shows the detailed view of "FBCENC Overview" dashboard. The dashboard layout is divided into four individual windows.

\section{Fairshare Window}

The top-left window shows the Fairshare allocations for different branches/counties. Fairshare is the primary food allocation policy used by food banks to distribute the food to each county based on the poverty rate. The Pound per Person in Poverty (PPIP)/Meals per Person in poverty (MPIN) is calculated by dividing the amount of food distributed to the people in poverty. Different data mining models were built to evaluate whether the fairshare policy being "met" or "unmet." The PPIP/ MPIN threshold of $75 \%$ is shown using a dotted line. Branches/counties meeting or exceeding this $75 \%$ threshold are shown in blue color and the unmet PPIP/MPIN allocations are shown in orange color.

\section{Donation by Year Window}

The bottom-left window shows the donation by year to forecast future donations. Data is presented using line chart for different years for all branches. Each branch can be selected using the multiple dropdown box to see donation received for a branch. Exponential smoothing method is used to forecast the donation data.

\section{Donation by Program Window}

Donation Program details are shown in the top-right window by gross weight using a stacked bar chart. When a particular branch is selected in a donation by program window all the participating program for that branch are updated in this window.

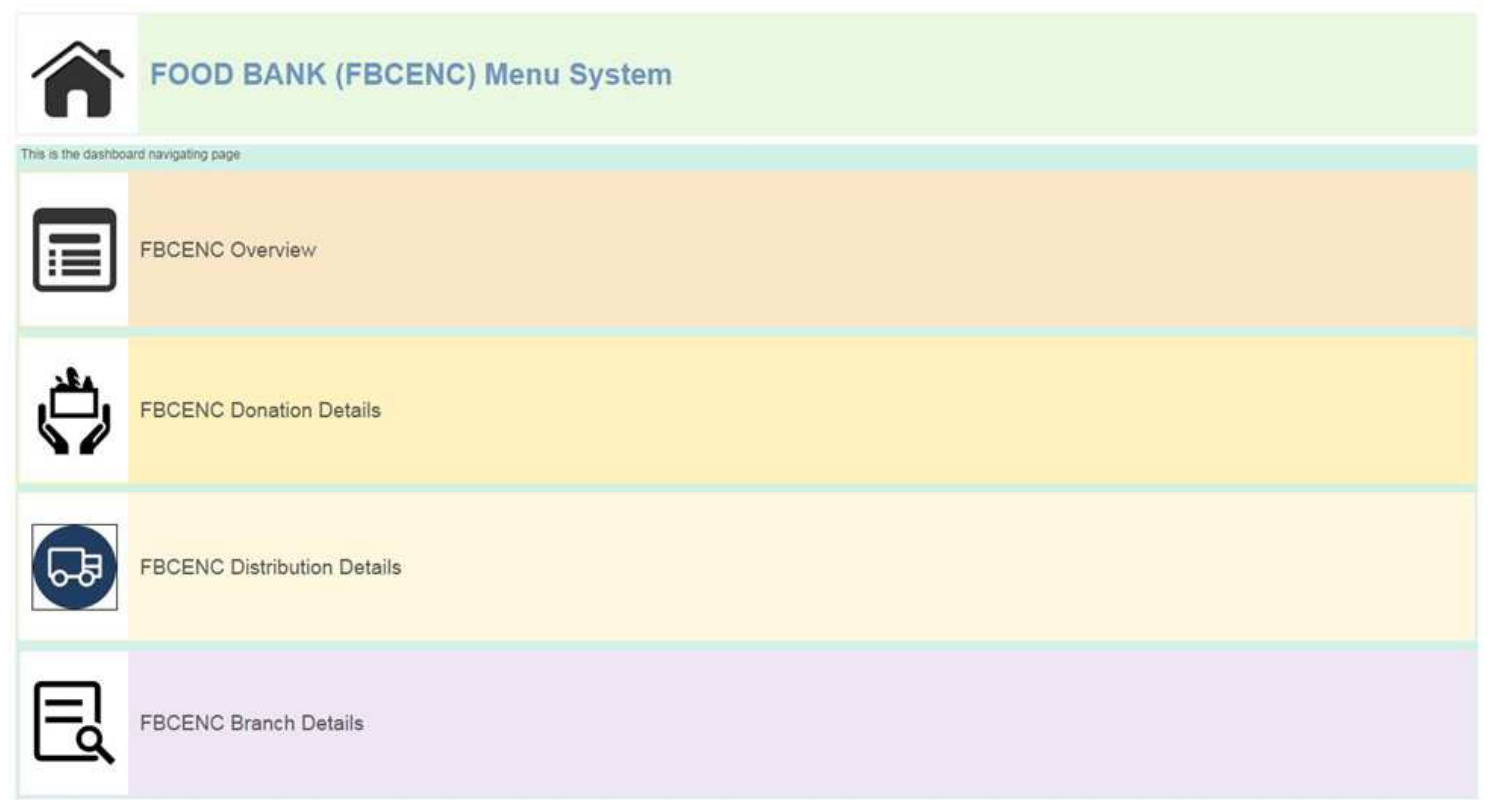

Fig. 3. Home page for FBCENC Dashboard 


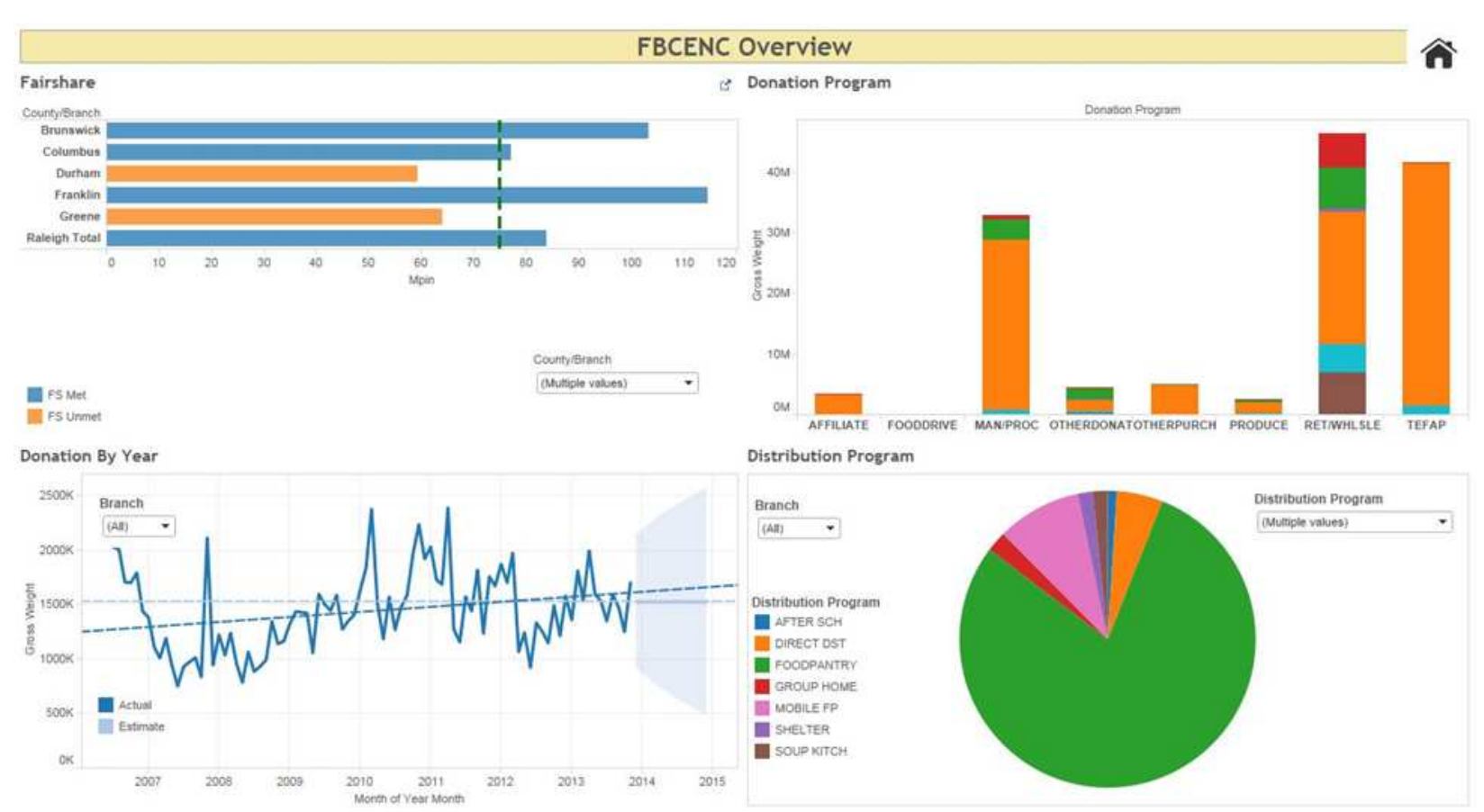

Fig. 4. FBCENC overview dashboard

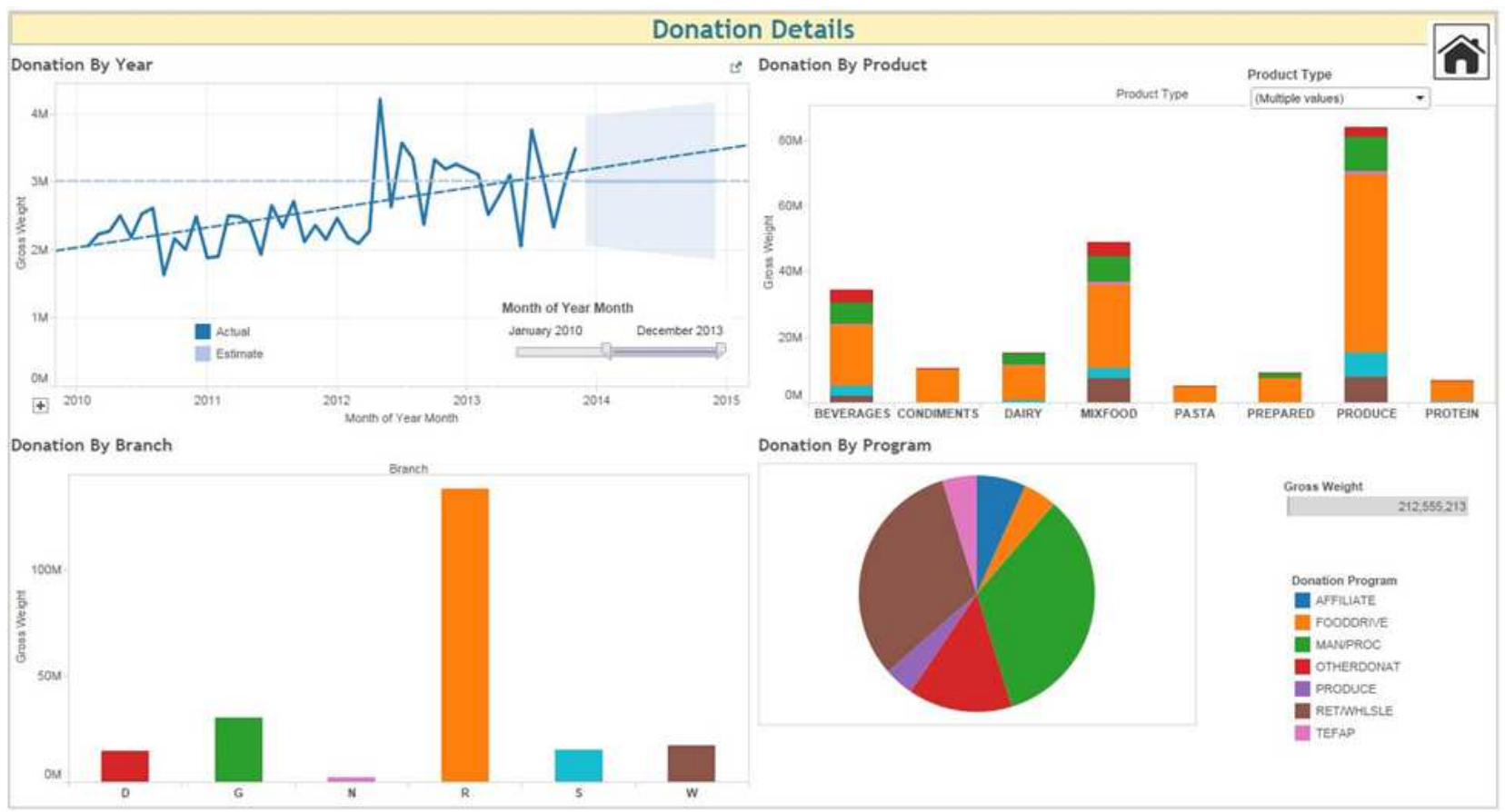

Fig. 5. FBCENC donation details dashboard

\section{Distribution Program Window}

A piechart shows the distribution program details using multi-color display. A multiple dropdown menu can be used for each of the above windows to select a specific branch.

\section{FBCENC Donation Details Dashboard}

Figure 5 shows the detailed view of "FBCENC Donation Details" dashboard. The dashboard layout is divided into four individual windows which gives details of the donation received through different donation programs. 


\section{Donation by Year Window}

The top-left window shows the donation by year to forecast future donations. Data is presented using line chart for different years. The number of years can be selected using a slider bar interactivity mode. Exponential smoothing method is used to forecast the donation data.

\section{Donation by Branch Window}

The bottom-left window shows the donation received in gross weight for all the branches. Data is presented using a bar chart for different branches.

\section{Donation by Product Window}

Donation Product details are shown in the top-right window by gross weight using a stacked bar chart. Multiple drop- = down box can be used to select different product types received.

\section{Donation by Program Window}

A piechart shows the donation by program details using multi-color display. When a particular donation program is selected using a click then all the other three windows are updated with information related to that donation program.

\section{SME Dashboard Evaluations}

The dashboards developed in this research were evaluated by Subject Matter Experts (SME) at the FBCENC. Five participants participated in the evaluation experiment. The PSSUQ survey results are as shown in Fig. 6. It shows the SME mean scores (error bars-standard deviation) for the three dashboard evaluation measures.

\section{Basic Statistics}

Data on time and overall satisfaction was collected and the basic statistics can be seen in the Table 1. The SME performed a list of tasks using both the traditional (existing) system and the new dashboard developed in this research. The mean time to generate a report for a particular branch using the traditional method was 16 minutes. Whereas, it took only 1.84 min using the new dashboard system. Thus, there was a significant reduction in the time as well as the number of steps to generate a report using the newly developed dashboards. This is evident from the task analysis shown in Fig. 1 and 2 that indicates a simplified report generation process using the new dashboard system.

Table 1. Basic statistics for SME evaluation

\begin{tabular}{|c|c|c|c|c|c|}
\hline & \multirow[b]{2}{*}{$\mathrm{n}$} & \multicolumn{2}{|c|}{ Overall Satisfaction } & \multicolumn{2}{|c|}{ Time (minutes) } \\
\hline & & Mean & StDev & Mean & StDev \\
\hline Old system & 5 & 3.0 & 0.71 & 16.0 & 4.24 \\
\hline New system & 5 & 6.6 & 0.55 & 1.84 & 0.25 \\
\hline
\end{tabular}

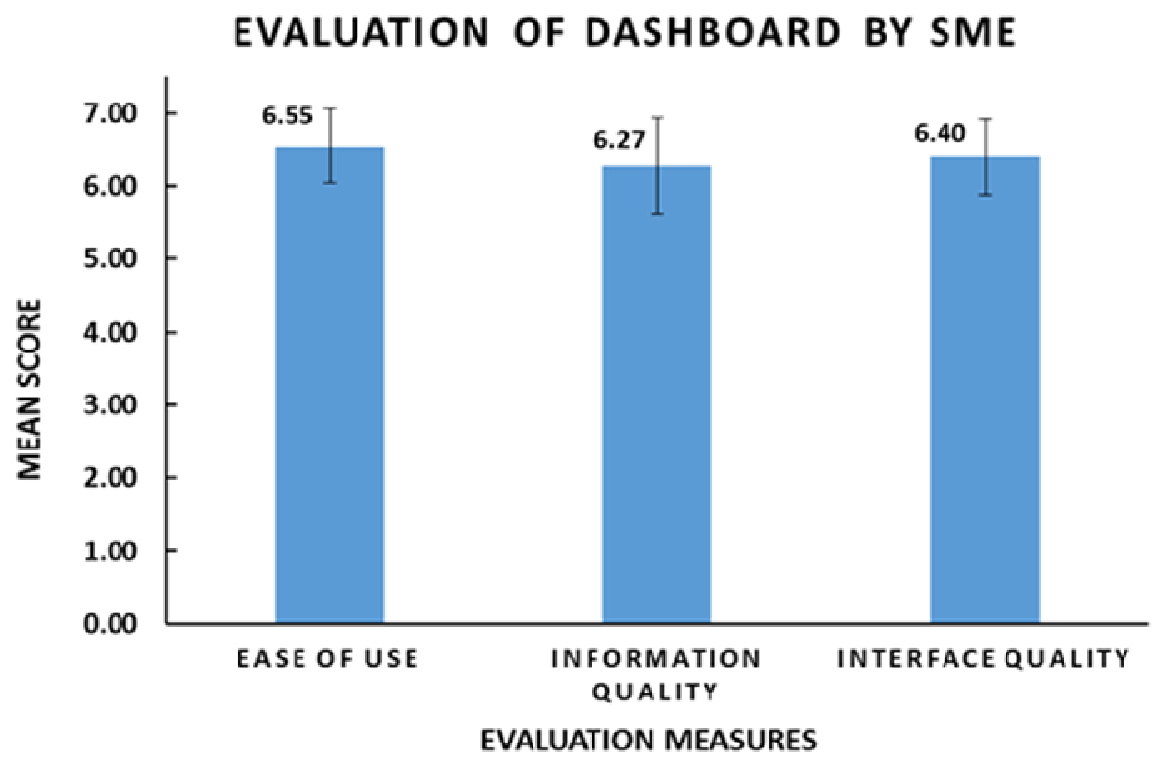

Fig. 6. Subject Matter Expert (SME) evaluation of dashboard 


\section{Inferential Statistics}

A correlation analysis between (a) time to completion and (b) overall satisfaction was performed. A strong negative correlation between the two dependent variables was found $(\mathrm{r}=-0.849, \mathrm{p}=$ 0.002). Therefore, the Hotelling's $\mathrm{T}^{2}$ test was conducted to analyze both time and satisfaction simultaneously. Model adequacy was checked to evaluate multi-variate normality for the variables and no violation was found. The output revealed that a statistically significant difference was found between the two systems when time and overall satisfaction were analyzed simultaneously (Wilk's $\Lambda=0.0339, \mathrm{~F}_{2,7}$ $=99.71, \mathrm{p}<0.0001)$.

A paired t-test was performed on the time and overall satisfaction separately. Results indicated a significant system effect on time $\left(\mathrm{t}_{4}=-7.89, \mathrm{p}<0.001\right)$ as well as overall satisfaction $\left(\mathrm{t}_{4}=-14.70, \mathrm{p}<0.001\right)$. Specifically, the new dashboard system had statistically the lowest mean time and the highest overall satisfaction score. Based on the above results it was concluded that the new dashboard system can serve the FBCENC for effective decision making.

The SME comments and evaluations were taken into consideration for improving the dashboard design. The recommendation of the SME included: (a) Color coding of different branches should match the FBCENC system (for example data for $\mathrm{R}$ branch should be displayed in purple color), (b) highlight each window title in upper case letters, (c) the multiple drop-down menus should be in the right edge of the window as applicable. These suggestions were included in the dashboard design for evaluation by participants to test overall satisfaction of the dashboard interface.

\section{Discussion}

\section{Overall Dashboard Evaluation}

Based on the inputs from the SME and feedback from the user participants an improved dashboard design was developed as shown in Fig. 7. The improvements in the dashboard design include: (a) Color display of different branches was matched with the existing FBCENC color code system (for example data for $\mathrm{R}$ branch is displayed in purple color), (b) highlighted each window title in upper case letters and (c) multiple drop-down menus placed in the right edge of the window as applicable. The new dashboard design was further evaluated for overall satisfaction.

Figure 8 shows the mean scores and standard deviation for the three dashboard evaluation measures of the PSSUQ survey. Based on the survey results, all three evaluation measures had a high mean score (close to 6.0) indicating that the interactive dashboard design was userfriendly and could capture food bank information. As can be seen from Fig. 8, the information quality had the highest mean score of 6.22 which indicated that the information displayed was well presented and participant could interpret the data for the given task.

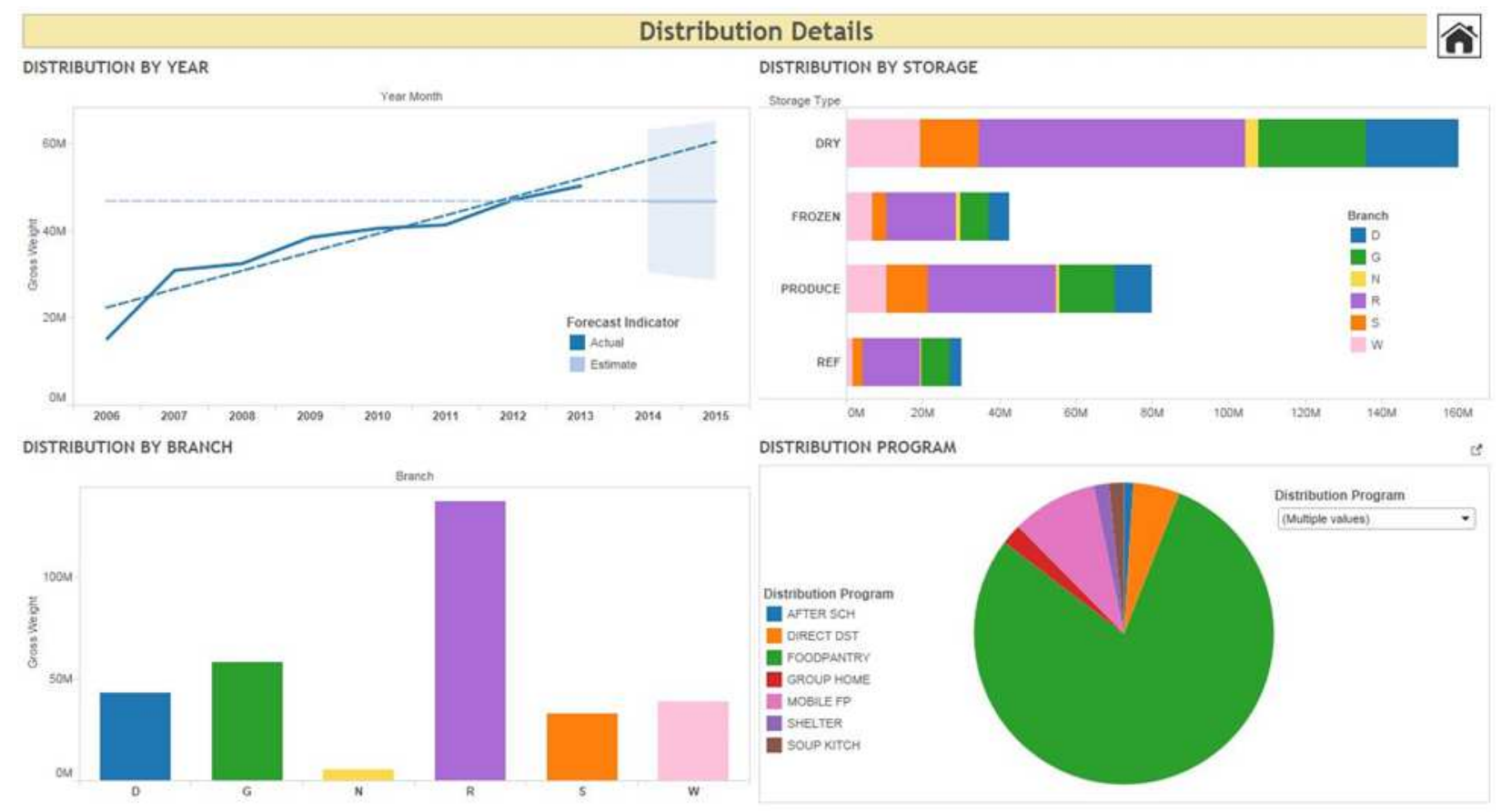

Fig. 7. Updated dashboard design for branch details 


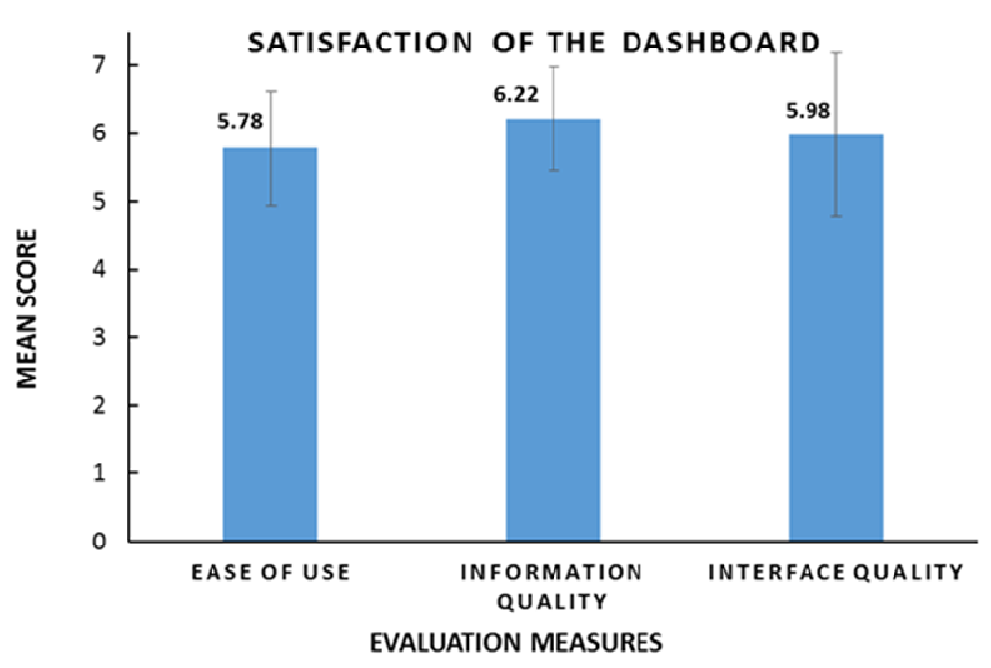

Fig. 8. Final evaluation of the dashboard

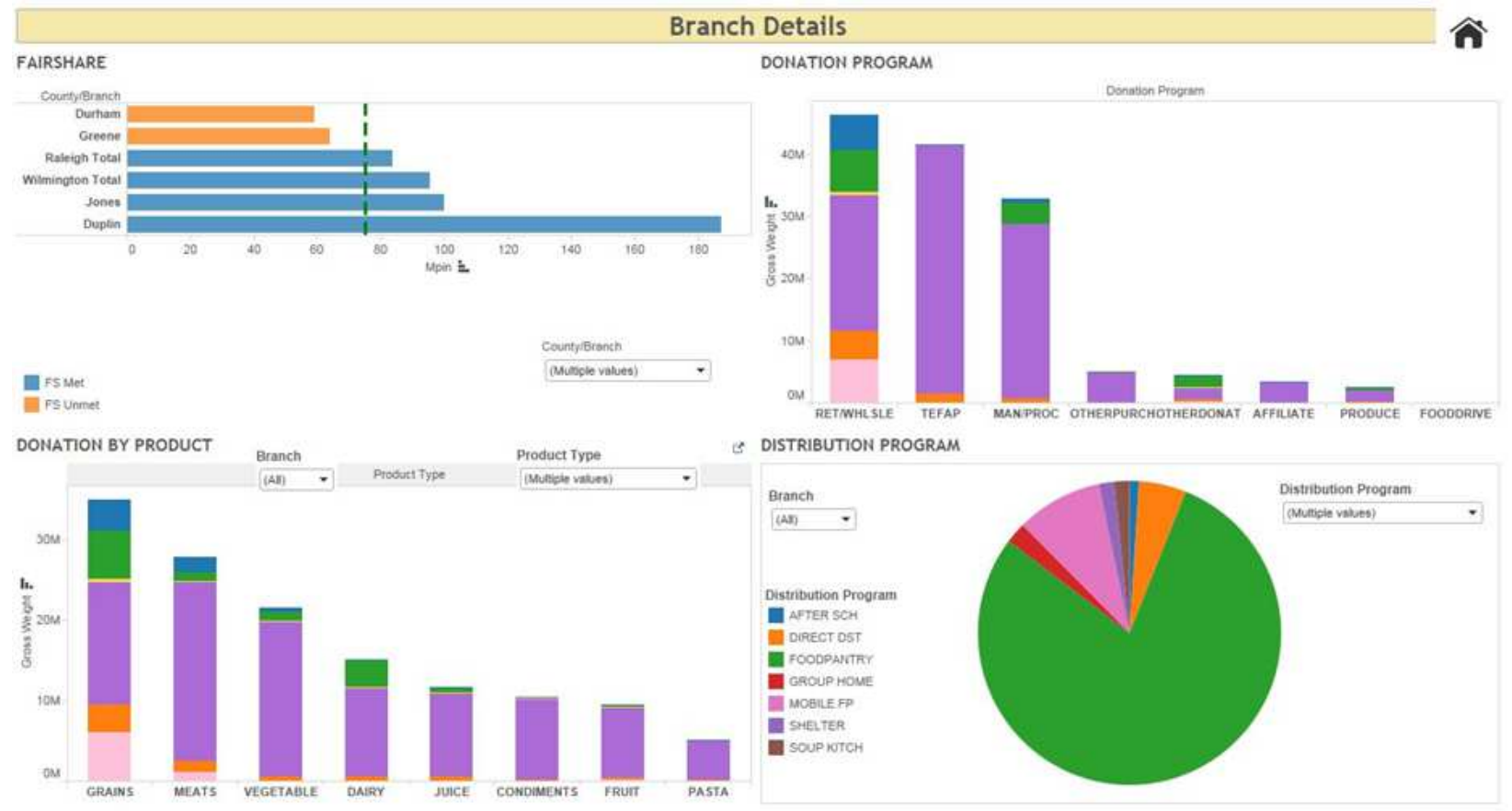

Fig. 9. Final interactive dashboard interface

The recommendations included that the bar graphs used in the dashboard to be made descending where applicable which will make it easy to compare between the data sets. Also, a few participants who were not familiar with FBCENC suggested of the color change for the charts, but due to the color coding of the branches this change was not possible. The final dashboard interface was developed based on the survey results and recommendations of the participants. The final dashboard interface for the Branch Details is displayed in Fig. 9.

\section{Conclusion}

In this research, interactive dashboards were developed for effective decision making in a food bank. With this methodology food bank personnel, can get access to structured information for decision making at different operational levels which is not readily available. Different visualization tools (pie chart, line charts and bar charts) appropriate to the food bank data were selected. Pie chart were used to evaluate the donation sources as well as distribution programs. Line charts were used to compare the 
demand and supply of food throughout the year. Bar charts were used to display different food types, branch types and donation types.

Interactive dashboards were developed using a User Centered Design approach. Results indicated that the new interactive dashboard outperformed the old method for both the evaluation measures (Wilk's $\Lambda=$ $\left.0.0339, \mathrm{~F}_{2,7}=99.71, \mathrm{p}<0.0001\right)$. These findings along with the feedback from the SMEs were used to revise the dashboard. The revised dashboard was tested for overall performance to evaluate (a) the ease of use, (b) information quality and (c) interface quality. The dashboard was highly rated for all three evaluation measures. The mean score for the information quality (6.22) was the highest among all three which indicated that the information was well presented and participants could interpret the data for the given task. The final dashboard interface was developed based on comments from the user evaluation.

The interactive dashboard developed in this research will provide food bank personnel easy access to data, improve information reporting and customized data presentation view. The results of this research provide a foundation for using a visual analytics approach to support decision making in the food bank. It can be extended to other food banks and humanitarian relief agencies.

\section{Acknowledgement}

We would like to thank the local food bank FBCENC for their support and the data during this research.

\section{Author's Contributions}

Yogeeta Desai: Design concept, all data-analysis and contributed to the writing of the manuscript.

Steven Jiang: Contributed in design and concept layout of the paper and assisted with the data analysis and reviewing of the manuscript

Lauren Davis: Provided the access to the local food bank data and contributed in reviewing and improving the manuscript.

\section{Ethics}

The authors declare that there are not ethical issues that could arise after the publication of this study.

\section{References}

Annesley, T.M., 2010. Bars and pies make better desserts than figures. Clin. Chem., 56: 1394-1400. DOI: $10.1373 /$ clinchem.2010.152298
Arias-Hernandez, R. and B. Fisher, 2013. A qualitative methodology for the design of visual analytic tools for emergency operation centers. Proceedings of the 46th Hawaii International Conference on System Sciences, Jan. 7-10, IEEE Xplore Press, pp: 126-135. DOI: $10.1109 / \mathrm{HICSS} .2013 .57$

Adams, J.A., 2002. Critical considerations for humanrobot interface development. Proceedings of the AAAI Fall Symposium, (FS' 02), pp: 1-7.

Andrienko, G., N. Andrienko and U. Bartling, 2007. Visual analytics approach to user-controlled evacuation scheduling. Proceedings of the IEEE Symposium on Visual Analytics Science and Technology, Oct. 30-Nov. 1, IEEE Xplore Press, pp: 43-50. DOI: 10.1109/VAST.2007.4388995

Barrett, C.B., 2010. Measuring food insecurity. Science, 327: 825-828. DOI: 10.1126/science. 1182768

Bloom, J., 2011. American Wasteland: How America Throws Away Nearly half of its Food (and what we can do about it). 1st Edn., Da Capo Press, Cambridge, ISBN-10: 0738215287, pp: 366.

Briefel, R.R., J. Jacobson and L. Tiehen, 2003. The emergency food assistance system: Findings from the client survey. US Department of Agriculture, Economic Research Service.

Brock, L.G. and L.B. Davis, 2015. Estimating available supermarket commodities for food bank collection in the absence of information. Expert Syst. Applic., 42: 3450-3461. DOI: 10.1016/j.eswa.2014.11.068

Coleman-Jensen, A., M. Rabbit, C. Gregory and A. Singh, 2015. Statistical supplement to household food security in the United States in 2014. USDA, Economic Research Service, AP-069.

Coleman-Jensen, A., M. Nord, M. Andrews and S. Carlson, 2011. United States Department of Agriculture. "Household Food Security in the United States in 2010."

Gundersen, C., B. Kreider and J. Pepper, 2011. The economics of food insecurity in the United States. Applied Econ. Perspect. Policy, 33: 281-303. DOI: $10.1093 /$ aepp/ppr022

Jonnalagadda, R., E.R. Walrond, S. Hariharan, M. Walrond and C. Prasad, 2005. Evaluation of the reasons for cancellations and delays of surgical procedures in a developing country. Int. J. Clin. Pract., 59: 716-720. DOI: $10.1111 /$ j.1742-1241.2004.00354.x

Keim, D., G. Andrienko, J.D. Fekete, C. Görg and J. Kohlhammer et al., 2008. Visual Analytics: Definition, Process and Hallenges. In: Information Visualization: Human-Centered Issues and Perspectives, Kerren, A., J. Stasko, J.D. Fekete and C. North (Eds.), Springer Berlin Heidelberg, New York, ISBN-10: 354070955X, pp: 154-175. 
Okore-Hanson, A., 2012. Empirical modeling of food demand and characterizing donor behavior during a natural disaster. $\mathrm{PhD}$ Thesis, North Carolina Agricultural and Technical State University.

Nuamah, I., L. Davis, S. Jiang and N. Lane, 2015. Predicting donations using a forecastingsimulation model. Proceedings of the Winter Simulation Conference, Dec. 6-9, IEEE Xplore Press, pp: 1880-1891.

DOI: $10.1109 /$ WSC.2015.7408305

SAS, 2015. SAS visual analytics.

Terry, J.R., 2013. Eliminating the shortage factor: Modeling the behavior of future donations using forecasting techniques to improve the distribution efficacy of a food bank. MSc Thesis, North Carolina Agricultural and Technical State University.
US, 2015. Usability survey.

Vredenberf, K., S. Isensee and C. Righi, 2001. UserCentered Design: An Integrated Approach. 1st Edn., Prentice Hall, Upper Saddle River, NJ., ISBN-10: 0130912956, pp: 288.

Wickens, C.D., J.D. Lee, Y. Liu and S. Gordon-Becker, 1998. An Introduction to Human Factors Engineering. 1st Edn., Longman, New York, ISBN-10: 0321012291, pp: 636. 